\title{
Inpatient Falls: Improving assessment, documentation, and management
}

\author{
Eleanor Nelson, Patrick Reynolds
}

Royal Devon and Exeter NHS Foundation Trust

\begin{abstract}
A frequently occurring job during on-call and out-of-hours shifts is reviewing a patient following a fall with this often being the responsibility of the most junior and inexperienced doctors. Following a pilot audit we identified inconsistencies in medical assessment and documentation, with $50 \%$ of expected data points not recorded.
\end{abstract}

Failure to complete a thorough assessment can lead to missed injuries, prolonged length of stay, and litigation. Using the plan, do, study, act (PDSA) cycle model this project sought to address this through providing teaching to junior doctors and the development of a pro-forma.

Three style cycles of data collection were performed; a formal baseline dataset, after delivering a teaching session to new junior doctors and following the trial of the new fall pro-forma. We selected 15 to 17 patient notes to review at random during a one month period for each data collection cycle and compared the medical assessment to the standards outlined by the National Patient Safety Agency (NPSA) guidelines.[1]

There were two key areas of improvement identified following the teaching session and introduction of the proforma. Documentation of a fall history was improved by nearly $30 \%$ being recorded in $100 \%$ of cases after the interventions. Documentation of a thorough musculoskeletal examination was improved from being recorded in just $54 \%$ of cases to $77 \%$ of cases; it was recorded in $100 \%$ of the cases where the proforma was used.

The project demonstrated the need to improve documentation and assessment of a patient who has fallen. Initial data collection has shown that assessment and documentation were improved providing teaching to junior doctors and by use of the document. The pro-forma has since been incorporated into hospital policy and now forms the compulsory documentation expected of the doctors and nurses managing patients following a fall. Ensuring easy access to the proforma and re-auditing after editing the document will be the next steps.

\section{Problem}

Inpatient falls are a growing problem with over 200,000 falls in hospitals each year.[1] A proportion of these have resulted in significant injuries including fractured hips and intracranial injuries.[1] Thorough assessment and documentation of the medical examination is vital to ensuring that medical problems and injuries are not missed, and also that appropriate handover to nursing staff and daytime medical staff can take place of any issues identified and of pending investigations.

While working as a junior doctor on call we realised that there was no guidance in place as to what a medical assessment of patient following a fall ought to entail, and no training to this end had been provided at induction. In addition we noted that there was the potential for disparity between falls assessments between doctors. This worryingly creates the potential for injuries to be missed, compromising patient safety. Word done to date demonstrates that a multi-disciplinary approach to managing in-patient falls decreases falls risk.[2, 3]

\section{Background}

The causes of a fall are multifactorial and complex, many inpatients in acute hospitals are at increased risk of having a fall during their stay. Risk of falling may be related to patient factors, environmental factors, and iatrogenic factors. Patient factors include inherent mobility or cognitive impairment, pre-existing medical conditions placing patients at increased falls risk such as syncope or postural hypotension, and factors such as patient footwear and appropriate use of walking aids. Environmental factors includes lighting within bays, obststacles, wet floors, and footwear. Latrogenic factors such as medications can also play a role in increasing patient fall risk.

Although prevention of a fall is paramount, clear understanding of what to do following a fall is essential. This assessment provides an opportunity to rapidly assess a patient for injuries and intervene to reduce potential harm from these injuries, as well as re-assessing their risk and implementing strategies to reduce further falls. A medical examination of a fall ought to be a thorough assessment that will elicit any neurological deficit or injury.[1] Relevant details about patient risk of fall and bleeding risk ought to be assessed and documented. If any imaging is required there ought to be a plan for the ward doctor to chase and act on these during normal working hours. Clear handover to the nurses must be provided along with details of frequency of observations and/or Glasgow Coma Scale (GCS) assessment if a head injury is present.

\section{Baseline measurement}

To demonstrate that there were deficiencies in medical 
documentation following inpatient falls at the Royal Devon and Exeter as a "proof of concept" step, we carried out an audit of inpatient falls and assessed whether particular details of the doctor's medical assessment were present or not.

Using the Datix reporting system we requested notes from any patient having an inpatient fall during the months of May and June 2014. A random snapshot of 17 sets of notes from these were selected. Using the NICE guidelines on falls assessment and with the guidance of one of the elderly care physicians we identified 16 data points that ought to have been recorded in the notes as part of the post-falls assessment. In total across all patients $50 \%$ of data points were found to be absent.

In order to capture a broad cohort of junior doctors we repeated the data collection on a similar number of patients at a time when the incoming group of newly qualified junior doctors had just started. Using the trusts incident reporting system we identified all patients who had fallen on a medical ward during a one month period. We then randomly selected 20 cases to review, we were able to view the medical notes of 17 of these. We scrutinised the documentation for these 17 cases recording the patient demographics, the time and circumstances of the fall, if they were reviewed by a doctor, and if so what was documented.

Despite the expectation that all patients should have basic observations recorded and be reviewed by a doctor following a fall we found that only $76 \%$ of cases were being reviewed by a doctor, $76 \%$ of cases had an initial set of observations recorded but only $35 \%$ had neurological observations documented. Of those seen by a doctor $31 \%$ did not have a fall history documented, $46 \%$ did not have documented evidence of a thorough musculoskeletal examination, and $77 \%$ did not comment whether the patient was anticoagulated or not.

See supplementary file: ds5845.pdf - "Sample of pro-forma developed"

\section{Design}

The baseline data collection demonstrated that not all junior doctors understood the importance of reviewing patients following a fall and there were clear discrepancies in the quality of documentation of this assessment.

We discussed with other junior doctors and felt that raising awareness of this problem among the new junior doctors and providing teaching and support at an early stage was a simple and potentially effective place to start. We therefore ran a teaching session within the first month of the new foundation year 1 doctors starting in the trust covering the expectations of them when a patient has fallen, as laid out in the Trusts Falls Policy, and what key components of their assessment are.

To improve the consistency of the approach to a patient and the documentation of this we felt the introduction of a pro-forma to guide and ideally speed up the documentation process would also be useful. We were aware that this has been implemented with success in other hospitals. On discussion with junior doctor colleagues we received positive feedback indicating that they would have found this useful when seeing patients after a fall, particularly out of hours.

\section{Strategy}

PDSA Cycle 0: Baseline data collection as described earlier.

PDSA cycle 1: In conjunction with the trusts lead clinician for falls we created a teaching session for the new foundation year one doctors within their first month of starting in the trust. We delivered this session at their compulsory protected teaching in conjuction with a Care of the Eldery Registrar. Following this we completed a further full cycle of data collection from the following months falls as identified using the trusts incident reporting system and then randomly selecting 20 cases to review. We were able to review the medical notes of 18 cases. We recorded the same data sets in this cycle as in the baseline data collection.

PDSA cycle 2: Following the teaching session, which received positive feedback but had a limited impact, we began the process of designing a pro-forma. We wanted to create a document to act as a guide to what should be included in the medical assessment but also to act as the documentation in the medical notes. We liaised the patient safety groups, lead nurses, matrons, falls steering groups, and lead clinicians during this process and had the document approved by the trusts documents committee. We then trialled this proforma on selected wards with the support of the lead nurses and ward matrons. We then carried out the data collection process as in the baseline data collection and cycle 1 for the months period where the pro-forma was trialled.

\section{Results}

At baseline it was found that for the cohort of patients assessed $76 \%$ of patients had initial observations performed, $35 \%$ had neurological observations performed, $76 \%$ of patients underwent medical review, $69 \%$ of patients had the fall history documented, $69 \%$ of patients were assessed for skeletal trauma, and $23 \%$ of patients had a review of their anticoagulation medication.

After cycle 1: Junior doctor teaching - 18 sets of notes were examined and it was found that $83 \%$ of patients had initial observations performed, $50 \%$ had neurological observations performed, $78 \%$ of patients underwent medical review, $100 \%$ of patients had the fall history documented, $64 \%$ of patients were assessed for skeletal trauma, and $14 \%$ of patients had a review of their anticoagulation medication.

After cycle 2: Introduction of the trial pro-forma - 17 patient notes were examined and it was found that $88 \%$ of patients had initial observations performed, $65 \%$ had neurological observations performed, $74 \%$ of patients underwent medical review, $100 \%$ of patients had the fall history documented, $77 \%$ of patients were assessed for skeletal trauma, and $38 \%$ of patients had a review of their anticoagulation medication. 
It should also be noted that out of this group of 17 patients the proforma was used only for four patients and for these patients $100 \%$ had initial observations performed, $100 \%$ had neurological observations performed, $100 \%$ of patients underwent medical review, $100 \%$ of patients had the fall history documented, $100 \%$ of patients were assessed for skeletal trauma, and $100 \%$ of patients had a review of their anticoagulation medication.

See supplementary file: ds5656.pptx - "Figure $1 \& 2$ showing the results after repeated cycles"

\section{Lessons and limitations}

The topic of inpatient hospital falls was quite an important one to address, however proved to be quite challenging in its execution. The data collection was relatively straightforward and the incident reporting system used in the trust is designed with data collection in mind. Sampling within a narrow time window such as a month gives a good idea of what is happening within a hospital at a particular time, however sampling in this way for this project will more likely capture the practice and documentation of a smaller number of junior doctors rather than the whole cohort within the trust at a given time. We acknowledged to allow for this given time pressures for data collection.

Once it came to the introduction of the proforma we ran into a few more difficulties. Patient safety in a hospital is deemed of the highest importance and looking to introduce new documentation addressing this can be a slow process. Documents need approval and need to meet the requirements and standards of those whose job it is to maintain those standards. We did receive a lot of support from senior staff at the hospital with regards to this and the document was generally well received.

One of the difficulties with introducing new documentation is making sure people know about it, making it available on the wards, making sure it gets used, and then gets topped up as routine. During the trial phase of the proforma only four out of 17 patients had the proforma used for their assessment. This process of implementing change on hospital wards takes time and has a lot of stakeholders whom it is important to involve earlier on. One of the potential stumbling blocks for our project leading to long term change is this very issue and future cycles as part of future projects ought to seek to address this.

\section{Conclusion}

We succeeded in our aims of identifying deficiencies in the assessment of patients who fall in hospital and associated documentation. Identifying this allowed us to proceed to implement change in hospital policy and standardise the documentation of the medical assessment of inpatient hospital falls.

Key areas of improvement identified were documentation of a fall history which was increased by nearly $30 \%$ being recorded in $100 \%$ of cases after the interventions. Documentation of a thorough musculoskeletal examination was increased from being recorded in just $54 \%$ of cases to $77 \%$ of cases; it was recorded in $100 \%$ of the cases where the proforma was used. Use of the proforma was associated with $100 \%$ compliance with the points outlined by the NPSA.

Sustaining this change in the future will be the main challenge and medical and nursing staff ought to be prompted to use the new document to allow it become business as usual for the wards. Standardised documentation will facilitate auditing falls in the future.

\section{References}

1. NPSA. Slips trips and falls in hospital data update. NPSA 2010.

2. Gibson R, Heaney A, Hull K. Can a multi-factorial assessment and interventional programme decrease inpatient falls in an elderly care ward? BMJ QIR 2013; 2 No.1.

3. Healey F, Monro A, Cockram A, Adams V, Heseltine D. Falls care plan - Using targeted risk factor reduction to prevent falls in older in-patients: a randomised controlled trial. Age Ageing $2004 \mathrm{Jul} ; 33(4): 390-5$.

\section{Declaration of interests}

The authors have no conflicts of interest to declare in this project.

\section{Acknowledgements}

The authors would like to thank Dr James Mulcahy and Dr Ray Sheridan for their guidance during this project.

\section{Ethical approval}

This work was intended to improve patient safety and care, and implement changes in Trust policy without carrying out interventions on human subjects. As such under Trust research policy at the Royal Devon and Exeter hospital it was exempt from ethical approval being an improvement study rather than a study on human subjects. All patient data was anonymised during analysis in line with trust information governance policies. 